\title{
PRUEBAS DE CAMPO PARA EVALUAR CALIDAD CALOSTRAL EN LAALPACA
}

\author{
Field Tests to Evaluate Colostrum Quality inAlpaca
}

\author{
Hanna Flodr ${ }^{1}$, Jane C. Wheeler ${ }^{2}$, Paloma Krüger D. ${ }^{2}$, Juan Olazábal L. ${ }^{3}$, \\ Raúl Rosadio A. ${ }^{2,3,4}$
}

\section{Resumen}

Las concentraciones de inmunoglobulinas (Igs) calostrales en la mayoría de especies productivas determinan los niveles de Igs en sus crías, y las fallas en la transferencia pasiva ocasionan susceptibilidades a infecciones en el recién nacido. El presente estudio evaluó dos pruebas de campo (grado de viscosidad visual y uso de refractómetro) para determinar la calidad del calostro de la alpaca en 77 muestras. Asimismo, se determinó la concentración de Igs mediante una prueba de inmunodifusión radial en 26 muestras de calostro y en 77 muestras de suero sanguíneo de crías obtenidas entre las 36 a 48 horas del nacimiento. Las muestras de calostro se analizaron visualmente para determinar grados de viscosidad (1 a 5), y con el refractómetro de azúcar Brix para determinar sólidos totales. El 60\% de las muestras calostrales presentó grados de 2-5 de viscosidad y lecturas promedio de $37.3 \%$ por el refractómetro de Brix, encontrándose una correlación altamente significativa entre viscosidad y lecturas por el refractómetro Brix $\left(\mathrm{p}<0.0001 ; \mathrm{r}^{2}=\right.$ 0.69). El IgG sérico en crías fue de $2679 \pm 603$ mg/dL y de IgG calostral fue de $28337 \pm 5593$ $\mathrm{mg} / \mathrm{dL}$, encontrándose un solo animal con fallas de transferencia pasiva (valores séricos: $750 \mathrm{mg} / \mathrm{dL}$ ). Las concentraciones de IgG calostrales tienen una correlación significativa con las lecturas del refractómetro ( $<<0.0007)$, no así con las concentraciones de IgG séricas de las crías $(\mathrm{p}=0.15)$. Similarmente, la correlación entre las lecturas de refractometría Brix y los valores séricos de IgG de crías fue baja ( $\mathrm{p}=0.338)$. La alta correlación entre la escala del \% Brix y las IgG calostrales muestran que el refractómetro de azúcar mide correctamente los niveles de IgG, por lo que sería un buen indicador de la calidad del calostro en condiciones de campo. Sin embargo, la baja correlación entre la escala de Brix y las IgG séricas de crías limita el valor de la utilización del refractómetro para predecir el estatus de transferencia pasiva en los neonatos.

Palabras clave: alpaca, inmunoglobulinas, falla de transferencia pasiva, refractometría Brix, calostro

${ }^{1}$ Royal Veterinary College of London, United Kingdom

${ }^{2}$ CONOPA - Instituto de Investigación y Desarrollo de Camélidos Sudamericanos, Lima, Perú

${ }^{3}$ Unidad de Biología y Genética Molecular, Facultad de Medicina Veterinaria, Universidad Nacional Mayor de San Marcos, Lima, Perú

${ }^{4}$ E-mail: rrosadio@gmail.com 
In the majority of livestock species, the concentration of maternal immunoglobulin (Ig) in colostrum determines the Ig level in their offspring, and the failure of passive transfer of maternal immunoglobulins results in their susceptibility to disease. This study evaluates two methods (visual assessment and Brix refratometry) for determining alpaca colostrum quality in the field. Evaluations of 26 fresh colostrum samples from 77 alpaca mothers were compared with the results obtained by radial immunodiffusion assay for 77 blood serum samples from 36-48 hours old offspring, and 26 colostrum samples. The colostrum was collected from the dams immediately postpartum and pre-suckling and blood was taken from the crias by jugular venipuncture. Grades of calostral viscosity were assessed visually, with 60\% at 2-5, and total calostral solids measured by Brix sugar refractometry averaged $37.3 \%$. The radial immunodiffusion test yielded newborn IgG serum levels of $2679 \pm 603.4 \mathrm{mg} / \mathrm{dL}$ and colostral IgG levels of $28337 \pm 5593 \mathrm{mg} / \mathrm{dL}$, and only one animal registered failure of immune transfer with serum levels of $750 \mathrm{mg} / \mathrm{dL}$. Results obtained by visual assessment of the colostrum coincide with those obtained by refractometry ( $p=0.0007)$, but differ from the serum IgG concentrations in the newborn animals ( $\mathrm{p}=0.15)$, as do the Brix refractometry readings $(\mathrm{p}=0.338)$. Due to the lack of low IgG levels in the colostrum samples, it was impossible to determine if a relationship exists between observed viscosity and colostral IgG concentration. Nonetheless, the positive correlation $(\mathrm{p}<0.001)$ between viscosity and Brix refractometry readings point to the need for further research.

Key words: alpaca, immunoglobulins, failure of passive transfer (FPT), Brix refractometry, colostrum

\section{INTRODUCCIÓN}

Los camélidos nacen con un sistema inmune bastante inmaduro por lo que se encuentran incapacitados para responder adecuadamente a agentes patógenos (Garmendia et al., 1987; Weaver et al., 2000a). Esto es consecuencia de la estructura física de la placenta, de tipo microcotiledonaria y epiteliocorial, que impide la transferencia de inmunoglubilinas (Igs) de la madre al feto (Bravo et al., 1997; Weaver et al., 2000a; Wernery, 2001). La protección inmunológica del neonato, durante las primeras semanas de vida, depende de la ingestión oportuna de calostro de buena calidad, así como de la eficiente permeabilidad intestinal durante las primeras horas de vida (Garmendia et al., 1987; Weaver et al., 2000a; Wernery, 2001). Desafortunadamente, la producción de calostro con altos niveles de anticuerpos, así como la adecuada ingestión por parte de la cría no siempre son las esperadas, desencadenándose la denominada falla de transferencia pasiva (FTP), con niveles de anticuerpos séricos menores a $1000 \mathrm{mg} / \mathrm{dl} \mathrm{a}$ las 48 horas del nacimiento (Garmendia et al., 1987; Garmendia y McGuire, 1987b; Weaver et al., 2000a; Wernery, 2001). La ocurrencia de FTP en alpacas neonatas ha sido detectadas en distintas áreas geográficas, con prevalencias de 9.0 a $20.5 \%$ (Garmendia et al., 1987; Weaver et al., 2000a,b), muchas veces asociada a una alta incidencia de enfermedades infecciosas (Lewis y Picut, 1989; Koterba et al., 1990; Mair et al, 1998; Wernery, 2001).

La FTP puede prevenirse con buenas prácticas de manejo, siempre y cuando se detecte oportunamente. Sin embargo, las metodologías disponibles para evaluar el estado de transferencia pasiva miden niveles 
de Igs sérica 36-48 horas después del nacimiento, momento en el cual la pared intestinal se encuentra incapacitada para absorber eficientemente las anticuerpos calostrales (Garmendia et al., 1987). La eficaz corrección de la FTP, en estas edades, es laboriosa e involucra transfusiones de plasma (20-40 $\mathrm{ml} / \mathrm{kg}$ de peso vivo) (Wernery, 2001). Por otro lado, la inmunodifusión radial, prueba definitiva para evaluar niveles de Igs dista mucho de ser un diagnóstico rápido para FTP en el campo, pues la prueba requiere una incubación de 24 horas (Drew y Fowler, 1995; Weaver et al., 2000a).

Se dispone de otros métodos de evaluación. Algunos miden proteínas totales del suero y las globulinas por el método de Biuret modificado, usando un analizador automatizado, mientras que otros métodos evalúan la turbidez con sales, tales como el sulfito de sodio. El uso de un refractómetro manual capaz de determinar los sólidos totales del suero muestran una correlación significativa con los niveles de IgG séricos, pero no existen datos significativos disponibles de sensibilidad y especificidad, imposibilitando considerar al refractómetro como prueba de uso clínico rutinario (Weaver et al., 2000a). Sin embargo, el mayor inconveniente en todos estos métodos, es que evaluan el estado de transferencia pasiva después de haber transcurrido el tiempo crucial para que la cría se pueda beneficiar de tratamientos profilácticos calostrales.

Por otro lado, existen numerosos estudios en especies domésticas, incluyendo el equino, que demuestran una correlación significativa entre la concentración de IgG y la gravedad específica calostral (Morris et al., 1985; Garmendia y McGuire, 1987; Koterba et al., 1990; Waelchi et al., 1990; LeBlanc et al., 1986, 1992; Chavatte et al., 1998; Tizard, 2000). Esta correlación ha permitido usar el refractómetro de azúcar para predecir el estado del calostro e identificar a potros en riesgo de padecer FTP. La refractometría calcula indirectamente los sólidos totales en una solución midiendo la luz de reflexión (Cash, 1995; Drew y Fowler, 1995). La escala Brix, por ejemplo, utiliza el índice de reflexión de la sucrosa pura en el agua, donde el índice de reflexión es de 1\% para este azúcar. Sin embargo, este refractómetro mide solamanente los sólidos totales sin poder diferenciar sus componentes (Cash, 1995; Drew y Fowler, 1995). No obstante, las Igs son los mayores componentes del calostro, de allí que resultaría un método rápido, barato y aplicable como una "prueba de campo" de aceptable sensibilidad, sobre todo para detectar calostro de baja calidad.

El propósito de este estudio fue evaluar la calidad del calostro mediante dos pruebas prácticas, la inspección visual de la viscosidad y el uso del refractómetro de azúcar Brix como métodos de predicción rápidos de los posibles estados de transferencia pasiva en crías de alpacas.

\section{Materiales y MÉTOdos}

\section{Animales y Muestras}

Se trabajó con 54 alpacas (madres con sus crías) de la estación experimental La Raya (Universidad Nacional del Altiplano), a 4200 msnm y con 23 de la zona de Macusani de la Rural Alianza EPS, a 4600 msnm. Las alpacas se mantuvieron en pastizales naturales, sin haber recibido tratamiento antiparasitario, suplementos alimenticios ni vacunación alguna. En la estación experimental La Raya se administra rutinariamente antibióticos (tetraciclinas) por vía oral durante los tres primeros días de vida a todas las crías.

Se colectaron 26 muestras de calostro y sangre de todas las alpacas madres y de sus crías ( $\mathrm{n}=77$ ). Las muestras de calostro y sus respectivas muestras de sangre fueron colectadas inmediatamente después del parto y antes de amamantar a la cría. El resto de las muestras de sangre se obtuvieron dentro de las 3-4 horas del parto. Se registró el nú- 
mero de parto (primeriza, multípara), y el peso y sexo de la cría.

La sangre se extrajo por punción de la vena yugular. El suero sanguíneo se extrajo por centrifugación y se almacenó a $-20^{\circ} \mathrm{C}$. Las muestras de sangre de Macusani, por falta de electricidad, fueron centrifugadas cinco días después del muestreo.

\section{Viscosidad}

Para determinar el grado visual de viscocidad, 3-4 gotas de calostro se colocaron entre los dedos índice y pulgar del evaluador. La viscosidad se interpretó mediante una escala arbitraria de 1-5, donde el valor de 1 fue para muestras de aspecto líquido, semejante a la leche acuosa y con muy baja o ninguna pegajosidad, y el valor de 5 para muestras de aspecto grueso, de movimiento lento, y con alto grado de pegajosidad. Todas las muestras fueron evaluadas por una sola persona, determinando subjetivamente valores intermedios.

\section{Sólidos Totales en Calostro}

Se tomó $1 \mathrm{ml}$ de la muestra de calostro y se vertió en el refractómetro de azúcar (090\% Brix, E-line 90 Bellingham \& Stanley, UK). El refractómetro no pudo calibrarse al $0 \%$ Brix, de modo que se empleó la escala de $2 \%$ Brix como lectura mínima, y se dedujo este valor en todas las muestras.

\section{Análisis de Laboratorio}

Las 77 muestras de sangre y 26 muestras de calostro fueron analizaron mediante el kit de inmunodifusión radial para IgG de camélidos (Triple J Farms, USA), siguiendo las instrucciones del fabricante.

En el caso del suero, se agregó $5 \mu \mathrm{l}$ de suero por pocillo en una placa de 24 pocillos conteniendo el anti-IgG de camélidos, alternando con similares cantidades de los tres sueros controles incluidos en el kit. La placa se incubó durante toda la noche a una tem- peratura entre 20 a $24{ }^{\circ} \mathrm{C}$. El diámetro de la zona de precipitación y de los controles se leyeron a las 24 horas. Se trazó una curva estándar con los datos de los controles y el diámetro resultante de las muestras fue comparado con la curva estándar. Los valores mayores a $9.4 \mathrm{~mm} /$ diámetro se encontraban fuera de la tabla de referencia y, por lo tanto, considerados como mayores de 3215 mg/dl; sin embargo, fueron asignados como valores iguales a $3215 \mathrm{mg} / \mathrm{dl}$.

En el caso del calostro, las muestras fueron diluidas 10 veces en solución salina (Bravo et al., 1997) antes de ser transferidas a la placa de inmunodifusión radial. Similarmente, se utilizaron estándares de concentraciones conocidas para comparar el IgG calostral. Todas las muestras con un diámetro mayor o igual a $8.5 \mathrm{~mm}$ fueron consideradas como concentraciones de IgG iguales o mayores de $32150 \mathrm{mg} / \mathrm{dl}$, pero fueron registradas como valores iguales a $32150 \mathrm{mg} / \mathrm{dl}$.

\section{Analisis Estadístico}

Se obtuvieron promedios y desviaciones estándares de concentraciones de IgG. Los datos fueron analizados usando modelos de correlaciones paramétricas y una prueba $\mathrm{T}$ no pareada. Los cálculos estadísticos fueron realizados utilizando el programa Prism con un valor de significancia (valor P) menor o igual a $5 \%$.

\section{Resultados}

El 40\% de las 77 muestras de calostro fueron de aspecto acuoso correspondiendo a grados 1-1.5, mientras que el $60 \%$ restante fueron de grados 2 a 5 . La lectura de las muestras con el refractómetro Brix dio un promedio porcentual de $37.3 \pm 4$, con rangos de 28 a 50\%. Se encontró una correlación altamente significativa entre la viscosidad del calostro y las lecturas del refractómetro $\left(p<0.0001 ; r^{2}=0.69\right)$ (Fig. 1a). No obstante, se tuvo varias muestras con grado de 
(A)

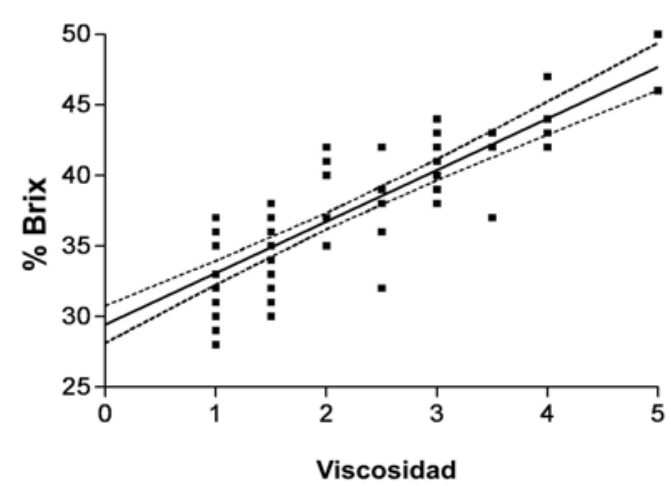

(B)

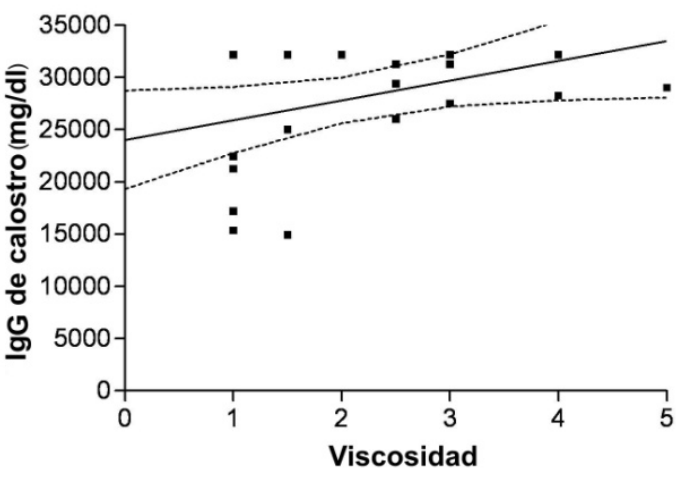

Figura1. Relación entre viscosidad y estimaciones de inmunoglobulinas en muestras de calostro de alpaca. A) Relación entre viscocidad y lecturas Brix; B) Relación entre viscosidad e IgG

(A)

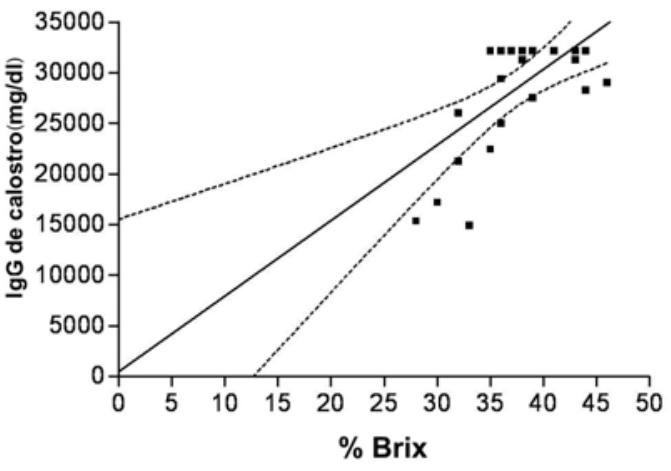

(B)

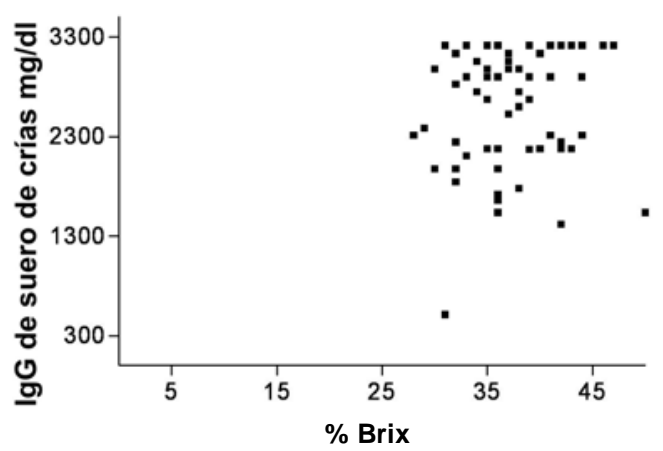

Figura 2. Relaciones entre concentraciones de IgG en calostro y suero de crías de alpaca con lecturas del refractómetro Brix. A) Relación entre IgG de calostro y lecturas Brix de calostro; B) Relación entre IgG de suero de crías y lecturas Brix de calostro. Todos los valores $>3215 \mathrm{mg} / \mathrm{dl}$ en suero o $>32150 \mathrm{mg} / \mathrm{dl}$ en calostro se registraron como 3215 y $32150 \mathrm{mg} / \mathrm{dl}$, respectivamente

viscocidad 1 que presentaron una gran variedad de concentraciones de Igs calostrales, aunque en general, las concentraciones de IgG mostraron una correlación significativa con el grado de viscocidad ( $p=0.044$ ) (Fig. 1b).

La concentración de IgG calostral fue de $28337 \pm 5593 \mathrm{mg} / \mathrm{dl}$, con rangos de 14 390 a mayores de $32150 \mathrm{mg} / \mathrm{dl}$ (superiores al límite máximo de detección). Las con- centraciones de IgG calostrales mostraron una alta correlación con las lecturas del refractómetro ( $\mathrm{p}=0.0007 ; \mathrm{r}^{2}=0.38$ ) (Fig. 2a); sin embargo, se observó una pobre correlación entre los niveles de IgG de suero de las crías con los valores Brix ( $\mathrm{p}=0.338$ ) (Fig. 2b), así como una baja correlación entre concentración IgG calostral y niveles en el suero de las crías ( $\mathrm{p}=0.15)$ (Fig 3). 


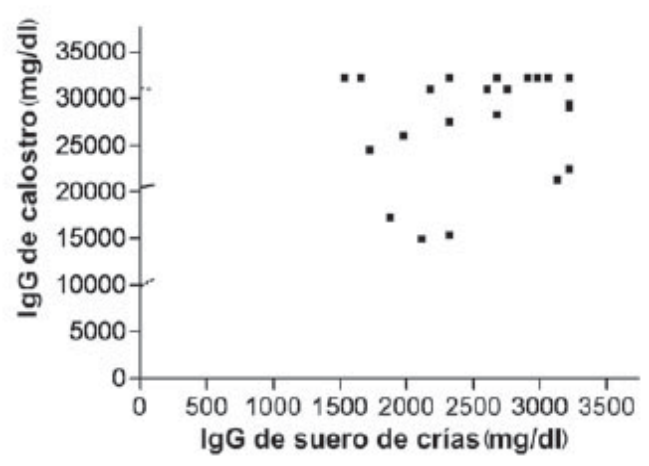

Figura 3. Relación entre concentración de IgG de suero y concentración de IgG de calostro en alpacas. Todos los valores $>3215 \mathrm{mg} / \mathrm{dl}$ en suero o $>32150 \mathrm{mg} / \mathrm{dl}$ en calostro se registraron como 3215 y $32150 \mathrm{mg} / \mathrm{dl}$, respectivamente

La concentración promedio de IgG séricas de las crías fue de $2679 \pm 603 \mathrm{mg} / \mathrm{dl}$, donde el 28.6\% (22/77) mantenían concentraciones superiores a $3215 \mathrm{mg} / \mathrm{dl}$ (registradas como $3215 \mathrm{mg} / \mathrm{dL}$ ) (Fig. 3). La prueba detectó solamente a una cría con una concentración sérica de $750 \mathrm{mg} / \mathrm{dl}$, lo cual es considerado como falla de transferencia pasiva.

No se encontraron diferencias significativas en las concentraciones de IgG séricas de las crías por efecto de la procedencia de los animales (La Raya y Macusani), sexo (hembra: 2766 mg/dl; macho: 2705 mg/dl), peso de la cría o número de parto de la madre (primerizas: 2667 mg/dl; multíparas: 2668 $\mathrm{mg} / \mathrm{dl}$ ). Tampoco se encontró diferencias significativas en las lecturas Brix por efecto de la procedencia de los animales (La Raya y Macusani) o del número de parto (primerizas y multíparas).

\section{Discusión}

La observación visual del calostro es aparentemente una buena alternativa como prueba rápida para evaluar su calidad. La alta correlación $(p<0.0001)$ entre el valor del refractómetro Brix y la viscosidad es sorprendente e interesante, dado que esta relación no es tan prominente en otras especies, con excepción de la yegua donde la densidad del calostro se considera como un medio de evaluación visual confiable (LeBlanc et al., 1986; Koterba et al., 1990; Mair et al., 1998).

Muestras de calostro con viscocidades de 1.5 no presentaron lecturas superiores a $40 \%$ Brix y aquellas con viscocidades de 3 no dieron lectura inferiores a 35\% Brix (Fig. 1a). Por otro lado, muestras de calostro con viscocidad mayor de 2 no presentaron concentraciones de IgG calostrales inferiores a $25000 \mathrm{mg} / \mathrm{dL}$ (Fig. 1b). Estos resultados sugieren que el refractómetero de azúcar puede ser útil para una determinación indirecta de las concentraciones de sólidos totales en el calostro. Desafortunadamente, en este estudio no se lograron detectar valores de IgG deficitarios en calostro ( $<10000 \mathrm{mg} / \mathrm{dl})$ (Weaver et al., 2000a), imposibilitando definir el umbral mínimo de calostro en la escala Brix e impidiendo evaluar adecuadamente a la viscosidad como método para detectar calostro de bajas calidades. Sin embargo, la estrecha relación entre viscosidad y lecturas del refractómetro dentro de los valores encontrados permite sugerir a la inspección vi- 
sual, a pesar de su subjectividad, como una prueba de campo rápida y efectiva para estimar la calidad del calostro.

Las muestras de calostro analizadas contienen suficiente cantidad de Igs que se tradujeron en concentraciones séricas adecuadas en las crías que ingerieron estos calostros. El nivel sérico de IgG (2679 mg/dl) en las crías a las 48 horas de edad son ligeramente altos comparados a los datos de Bravo et al. (1997) en animales de la misma zona geográfica, pero bastante mayores respecto a animales criados fuera del Perú (Weaver et al., 2000a,b). Por otro lado, una sola cría con nivel de IgG sérico de $750 \mathrm{mg} / \mathrm{dl}$ dentro de una población de 77 crías muestreadas representa una baja prevalencia comparada con los rangos de 9 a $20 \%$ reportados en la literatura (Garmendia et al., 1987; Weaver et al., 2000a).

Las crías de alpaca nacen agammaglobulinémicas, pero después de la ingesta de calostro se observan aumentos lineales de concentración de IgG de manera similar a lo que ocurre en otras especies domésticas (Drew y Fowler, 1995; Bravo et al., 1997). El factor determinante para alcanzar niveles protectivos de IgG séricas en las crías es la concentración de IgG presente en el calostro (Stott y Fellah, 1983; Morris et al., 1985; LeBlanc et al., 1986, 1992). Los resultados de este reporte, sin embargo, demuestran que en alpacas, al parecer, no existe una relación significativa entre la concentración de IgG de calostro de las madres y los niveles de IgG séricos de las crías. Esta baja correlación es consistente con otros resultados de la literatura (Garmendia et al., 1987). Desafortunadamente, al no haberse encontrado valores bajos de IgG en suero y en calostro, no se pudo determinar en forma precisa esta posible baja asociación.

La falta de correlación entre las IgG calostrales e IgG séricas de las crías sugieren, además de la concentración calostral, la existencia de otros factores que podrían ser importantes en la absorción de inmuno- globulinas en alpacas. A pesar de que se acepta que la capacidad de absorción de anticuerpos por el intestino es solamente por un periodo limitado, el tiempo de la primera ingesta de calostro en otras especies de animales domésticos es, asimismo, de gran importancia (Stott y Fellah, 1983; LeBlanc et al., 1992; Michanek y Ventorp, 1993). En terneros, la cantidad de IgG ingerida afecta el cierre del intestino de un modo lineal (Stott y Fellah, 1983; Michanek y Ventorp, 1993; Tizard, 2000). Las investigaciones realizadas en caballos demuestran que los anticuerpos calostrales se absorven activamente utilizando receptores Fc especializados que están presentes en las células epiteliales del intestino e ingresan al sistema circulatorio a través de los vasos quilíferos. Estas células, al ser remplazadas por células epiteliales maduras, 18 a 24 horas después del nacimiento, incapacitan la absorción intestinal de Igs (Morris et al., 1985; Lewis y Picut, 1989; Banks y McGuire, 1989; Koterba et al., 1990).

Niveles adecuados (>1000 mg/dl) de IgG séricas obtenidas en crías procedentes de madres con bajas concentraciones de Ig calostrales (Fig. 3), indicarían la existencia de un posible mecanismo de retroalimentación en esta especie animal que necesita ser elucidado. La fisiología exacta de la absorción de las inmunoglobulinas en crías de alpaca aún está por investigarse. Se desconoce, por ejemplo, el momento de cierre del intestino, pero el aumento lineal de la concentración de IgG séricas durante las primeras 24 horas seguido de un descenso a las 48 horas, evidencia la existencia de un patrón similar al de terneros con un tiempo de absorción óptimo entre las 0 y 12 horas de edad, tal como lo sugiere Garmendia et al. (1987).

La IgG, componente del 85\% de las proteínas del suero transferidas pasivamente a las crías, es la principal inmunoglobulina absorbida por el neonato. También se transfiere, aunque en menores cantidades, la IgM (Garmendia et al., 1987). Se desconoce, sin embargo, los isotipos de IgG preferentemente absorbidos, ya que las inmunoglobulinas de 
los camélidos son únicas y difieren dramáticamente en relación a otras especies de animales. En los camélidos, se conoce que más del 75\% de las proteínas séricas son moléculas de IgG carentes de cadena ligera y referidas como $\operatorname{IgG}_{2}$ e IgG Ig $_{3}$ y característicamente mucho más pequeñas (90 kDa) que los anticuerpos convencionales (150 kDa), con capacidades de mejor penetración tisular y una mejor biodistribución (Wernery, 2001). Estos anticuerpos, denominados microglobulinas, serían mucho más eficientes, por ejemplo, para neutralizar enzimas, que los anticuerpos convencionales. Se desconoce, todavía, los efectos biológicos de estas microglobulinas en la transferencia pasiva, ya que no se sabe que isotipos de IgG se encuentran presentes en calostro y, sobre todo, si estas microglobulinas se encuentran presentes en las secreciones calostrales.

Asimismo, los camélidos sudamericanos difieren de otras especies domésticas en la forma de producción del calostro. Investigaciones realizadas en la vaca, cerda, yegua y oveja han demostrado que el pasaje de las moléculas de IgG de la sangre hacia las glándulas mamarias ocurren momentos antes de la parición (Koterba et al., 1990; Tizard, 2000). En alpacas y llamas, los niveles de IgG séricos se mantienen constantes a través de toda la preñez, mientras que las concentraciones de IgG en la secreción mamaria se elevan 10 veces justo antes de la parición, para decaer dramáticamente después del nacimiento (Bravo et al., 1997). Esto sugiere una posible producción local de IgG calostral en la glándula mamaria que necesita ser elucidada.

Estudios realizados sobre el efecto de otros factores, como la edad de la madre y la parición, en otras especies domésticas arrojan resultados variables (Morris et al., 1985; LeBlanc et al., 1992; Erhard et al., 2001); sin embargo, un estudio en vacas determinó que la calidad del calostro mejora con el nú- mero de partos (Mohammed et al., 1988). Los resultados del presente estudio demuestran que no existe diferencia en las lecturas del refractómetro de azúcar en el calostro de madres multíparas y primerizas ni en los niveles respectivos de IgG de las crías. Debe indicarse, sin embargo, que durante el muestreo se observó que las madres multíparas tendían a producir una mayor cantidad de calostro que las madres primerizas. No se observó la cantidad de calostro ingerida, pero se sabe que una cría de 10 kg necesita consumir aproximadamente $100 \mathrm{ml}$ de calostro de calidad normal (22 $000 \mathrm{mg} / \mathrm{dL}$ ) para obtener niveles de IgG mayores a 1000 mg/dL (Wernery, 2001).

\section{Conclusiones}

- La alta correlación entre la escala del \% Brix y las IgG calostrales muestran que el refractómetro de azúcar mide correctamente los niveles de IgG por lo que sería un buen indicador de la calidad del calostro en condiciones de campo. Sin embargo, la baja correlación entre la escala de Brix y las IgG séricas de crías limita el valor de la utilización del refractómetro para predecir el estatus de transferencia pasiva en los neonatos.

- Se encontró una baja correlación entre la concentración de IgG calostrales de la madre e IgG séricas de las crías.

\section{Agradecimientos}

Este estudio fue parcialmente financiado por la beca Pequeños Proyectos y Viajes UFAW, Gran Bretaña y la Fundación Zebra (“Zebra Foundation”). Los autores expresan su gratitud a Brian Aldridge, Hugo Castillo Doloriert, Gustaf Rydevik y a todo el equipo de la estación experimental La Raya UNA y Rural Alianza, Macusani, Perú, por el apoyo brindado. 


\section{LiTERATURA CitADA}

1. Banks KL, McGuire TC. 1989. Neonatal immunology. In: Holliwel R, Gorman NT (ed). Veterinary clinical immunology. Philadelphia, Pennsylvania, USA: Saunders. p 117-145.

2. Bravo PW, Garnica J, Fowler ME. 1997. Immunoglobulin $G$ concentrations in periparturient llamas, alpacas and their crias. Small Ruminant Res 26: 145-149.

3. Cash RSG. 1995. Colostral quality determination by simple refractometry, a preliminary investigation. Centaur 11: 56-59.

4. Chavatte P, Clément F, Cash R, Grongnet JF. 1998. Field determination of colostrums quality by using a novel practical method. Proc Annual Convention AAEP 44: 206-209.

5. Drew M, Fowler ME. 1995. Comparison of methods for measuring serum immunoglobulin concentrations in neonatal llamas. J Am Vet Med Assoc 206: 1374-1380.

6. Erhard MH, Luft C, Remler HP, Stangassinger M. 2001. Assessment of colostral transfer and systemic availability of immunoglobulin $\mathrm{G}$ in newborn foals using a newly developed enzyme-linked immunosorbent assay (ELISA) system. J Anim Physiol Anim Nutr 85: 164-173.

7. Garmendia $A E$ Palmer GH, DeMartini JC, McGuire TC. 1987. Failure of passive immunoglobulin transfer: a major determinant of mortality in newborn alpacas. Am J Vet Res 48: 1472-1476.

8. Garmendia AE, McGuire TC. 1987. Mechanism and isotypes involved in passive immunoglobulin transfer to the newborn alpaca (Lama pacos). Am J Vet Res 48: 1465-1471.

9. Koterba AM, Drummond WH, Kosch PC. 1990. Immunological considerations. Equine clinical neonatology. Philadelphia, Pennsylvania, USA: Lea \& Febiger. 867 p.
10. LeBlanc MM, McLaurin B, Boswell R. 1986. Relationships among serum immunoglobulin concentration in foals, colostral specific gravity and colostral immunoglobulin concentration. J Am Vet Med Assoc 189: 57-60.

11. LeBlanc MM, Tran T, Baldwin JC, Pritchard EL. 1992. Factors that influence passive transfer of immunoglobulins in foals. J Am Vet Med Assoc 200: 179-183.

12. Lewis RM, Picut CA. 1989. Veterinary clinical immunology. Philadelphia, Pennsylvania, USA: Lea \& Febiger. 280 p.

13. Mair T, Love S, Schumacher J, Watson E. 1998. Equine medicine, surgery \& reproduction. Philadelphia, Pennsylvania, USA: Saunders. 498 p.

14. Michanek P, Ventorp M. 1993. Passive immunization of new-born dairy calves on three farms with different housing systems. Swed J Agr Res 23: 37-43.

15. Mohammed HO, Sherer J, Brennneman J. 1988. Factors associated with the levels of immunoglobulins in colostrum. Acta Vet Scand (Suppl) 84: 119-121.

16. Morris DD, Meirs DA, Merryman GS. 1985. Passive transfer failure in horses: incidence and causative factors on a breeding farm. Am J Vet Res 46: 22942299.

17. Stott GH, Fellah A. 1983. Colostral immunoglobulin absorption linearly related to concentration for calves. J Dairy Sci 66: 1319-1328.

18. Tizard. 2000. Veterinary immunology. $6^{\text {th }}$ ed. Philadelphia, Pennsylvania, USA: Saunders. 482 p.

19. Waelchi RO, Hässig M, Eggenberger E, Nussbaumer M. 1990. Relationships of total protein, specific gravity, viscosity, refractive index and latex agglutination to immunoglobulin $\mathrm{G}$ concentration in mare colostrum. Equine Vet J 22: 39-42.

20. Weaver DM, Tyler JW, Marion RS, Wallace LM, Nagy JK, Holle JM. 2000a. Evaluation of assays for determination of passive transfer status in neonatal llamas and alpacas. J Am Vet Med Assoc 216: 559-563. 
21. Weaver DM, Tyler JW, Scott MA, Wallace LM, Marion RS, Holle JM. 2000b. Passive transfer of colostral immunoglobulin $\mathrm{G}$ in neonatal llamas and alpacas. Am J Vet Res 61: 738-741.
22. Wernery U. 2001. Camelid immunoglobulins and their importance for the newborn - a review. J Vet Med B 48: 561-568. 